\title{
Flank Pain, CTCAE
}

National Cancer Institute

\section{Source}

National Cancer Institute. Flank Pain, CT CAE. NCI Thesaurus. Code C143488.

A disorder characterized by a sensation of marked discomfort on the lateral side of the body in the region below the ribs and above the hip. 